\title{
Bilateral Sagittal Split Osteotomy And Genioplasty - A Case Report.
}

\author{
Dr. Ajay Mohite ${ }^{1}$,Dr. Rajesh Kshirsagar ${ }^{2}$,Dr. Amod Patankar ${ }^{3}$, \\ Dr. Sudhir Pawar ${ }^{4}$, Dr. Vikram Singh ${ }^{5}$ \\ ${ }^{I}$ (Resident, Dept. of Oral \& Maxillofacial Surgery/Bharati Vidyapeeth University/India) \\ ${ }_{2}^{2}$ (Head of Dept. Dept. of Oral \& Maxillofacial Surgery/Bharati Vidyapeeth University/India) \\ ${ }^{3}$ (Associate Professor, Dept. of Oral \& Maxillofacial Surgery/Bharati Vidyapeeth University/India) \\ ${ }^{4}$ (Lecturer, Dept. of Oral \& Maxillofacial Surgery/Bharati Vidyapeeth University/India) \\ ${ }^{5}$ (Associate Professor, Dept. of Oral \& Maxillofacial Surgery/Bharati Vidyapeeth University/India)
}

\begin{abstract}
Bilateral Sagittal Split Osteotomy is a cosmetic surgical procedure for the correction of lower jaw deformities. It is accomplished through transoral incision. The osteotomy splits the ramus and the posterior body of the mandible sagittally, which allow either setback, advancement or rotation.
\end{abstract}

Keywords: Mandibular deformities, Complications, Advantages, Transoral incision, Chin deformities.

\section{Introduction}

Bilateral sagittal split osteotomy (BSSO) is a widely used orthognathic surgical technique for the correction of mandibular deformities. The main purpose of this elective procedure is the establishment of a class I occlusion with good function. Surgeons should also aim to establish a harmonious maxillofacial profile with good facial esthetics [1].

\section{Case Report}

A 25yrs male came to Bharati Vidyapeeth Dental College and Hospital, Pune with complain of forwardly placed upper front teeth \& abnormal chin.

On Extra-oral examination, Face type was mesofacial symmetry, Profile was convex, Maxilla was normal, Mandible was retrusive, Nose was average and Nasolabial angle was obtuse.

On Intra-oral examination, Incisor relationship was Class II Div 1, Canine relationship was Class II End on, Molar relationship was Class II End on, Maxillary \& Mandibular incisors was proclined, Overjet was $11 \mathrm{~mm}$, Overbite was $6 \mathrm{~mm}, \mathrm{TMJ}$ was Normal, Mandibular path of closure was upward, forward \& backward, Maximum inter-incisal opening was $40 \mathrm{~mm}$. Freeway space was $2.5 \mathrm{~mm}$, Breathing pattern was nasal, Tongue thrust was present and Circum oral muscles was normal during function.

Problem list was recorded as Skeletal CL II / Retrognathic mandible, Recessive chin, Proclined upper incisors, Proclined lower incisors and Increased over jet and over bite, Lower lip trap and tongue thrust.

The treatment plan was decided as Orthodontic correction of Proclined Upper Incisors, Proclined Lower Incisors, Increased over jet and over bite, Lower lip trap, Tongue thrust and Surgical correction of Skeletal CL II / Retrognathic mandible recessive Chin by Bilateral sagittal split osteotomy and Genioplasty.

\section{Surgical Procedure}

A bite block is inserted on the opposite side in between the upper and lower teeth for making easy access and projecting the ramus anteriorly. An incision is made on the lateral aspect of the anterior border of the ramus, overlying the external oblique ridge, from midway up the ascending ramus (to avoid buccal pad of fat) downward into vestibular depth till mandibular first molar region. The soft tissue dissection is started subperiosteally along the anterior border upwards, towards the coronoid process. Medially the soft tissues are reflected, until the lingula and the inferior alveolar nerve bundle and mandibular foramen is identified. The medial soft tissue dissection is stopped slightly posterior and superior to the lingula. While the medial soft tissues are being retracted, the medial cut is made through only the lingual cortex about $2 \mathrm{~mm}$ above the neurovascular bundle and just posterior to the lingula.

Following the completion of horizontal medial osteotomy, cut is then carried down the lateral most aspect of the anterior border of the ascending ramus to the region of the second molar. This osteotomy is made parallel to the lateral cortex. The bite block is removed and the periosteum from the lateral aspect of the mandible is elevated in the molar area till the inferior border of the mandible. For setback procedure, the vertical osteotomy cut is taken lateral to second molar, however, in major advancement cases, vertical cut is placed forward in the region of the premolar. Vertical cut is completed through the lateral cortex only. The cut extends 
through both the cortical plates at the inferior border of the mandible. With osteotome, all bony cuts are checked for their completeness. Two osteotomes are then inserted and used as levers to separate the segments. Spreader can be used to finally separate the segments. The same procedure is repeated on the other side. In case of advancement, the bony interferences should be checked and the distal fragment is advanced and locked into desired occlusion. The fixation of the fragments can be done by intraosseous wiring or lag screws or by bone plates.

In case of setback, the distal fragment is pushed back. There will be overlap of the proximal fragment in the molar region. The excess bone on the proximal fragment is cut off by taking another vertical cut, distal to the previous cut, including only proximal fragment. The fragment will be checked now for purpose approximation and fixation is carried out. The procedure gives excellent stable results. The obvious problem of this procedure is the close proximity of the osteotomy lines and the neurovascular canal. Care should be taken not to damage the inferior alveolar nerve during this procedure.

Plaster of Paris models from the maxilla and mandible are taken and the actual centric occlusion of the patient is recorded. The models are oriented in a semi adjustable articulator after face bow transfer. The models allow analyzing the: occlusion, shape of the dental arches, position, size and shape of the teeth, position of the jaws in relation to the skull base.

The procedure starts with three corticotomies. The first cut is made through the lingual cortex a few $\mathrm{mm}$ above the mandibular foramen parallel to the occlusion. The corticotomy is extended from the anterior to the posterior borders of the ramus. The second corticotomy is made through the buccal cortex in a vertical direction at the level of the first or second molar. The third corticotomy connects the first two osteotomy lines along the anterior border of the ascending ramus. The final split is completed with a thin osteotome, splitting the entire ascending ramus from the anterior to the posterior border of the ramus. A special bone spreader can be used to mobilize the segments. After the bilateral split is completed the large tooth bearing segment can be moved three dimensionally. Mandibulo-maxillary fixation is performed to position the large tooth bearing segment to the desired relationship with the maxilla. A prefabricated surgical splint (or wafer) may be used to facilitate this.

Some movements will require additional osteotomies or removal of bone to allow for a good alignment of the respective segments The Obwegeser osteotomy is a bilateral sagittal split osteotomy of the ramus, which divides the mandible into two smaller condyle bearing segments and a large segment consisting of the mandibular body including the teeth and chin.

Dal Pont changed the lower horizontal cut to a vertical cut on the buccal cortex between the first and the second molars, thereby obtaining broader contact surfaces and requiring minimal muscular displacement with improved access. The Hunsuck modification is the Obwegeser / Dal Pont osteotomy where the split does not extend to the posterior border of ramus.

For Genioplasty, the horizontal osteotomy at the symphysis is often done in conjunction with other major osteotomies and, thus, is frequently accomplished under a general anesthetic. However, it can be performed as a separate procedure on an outpatient basis under sedation and local anesthesia.

The mucosal incision is made on the labial side of the vestibule at about $1 \mathrm{~cm}$ above its depth and extends posteriorly to the first bicuspids. This incision is carried just below the mucosa to the depth of the vestibule and then angled directly to the labial cortex through the mentalis muscle. Periosteum is elevated inferiorly to a point just below the intended level of osteotomy. Laterally the periosteum is elevated to the mental foramen and then extended posteroinferiorly to the inferior mandibular border. The extent of the posterior cortical exposure is generally determined by the position of the mental foramen and the vertical height of the mandible in this area. No attempt is generally made to expose the mental nerve by releasing the soft tissue around it, primarily because the nerve can be small and friable, making inadvertent severing possible. It is helpful at this point to inscribe a vertical mark (or marks) into the bone across the planned osteotomy site so that the transverse position of the inferior fragment can be more easily oriented after the osteotomy.

The osteotomy cut is then made with a reciprocating saw. The length and angle of the horizontal cut can have profound effects on postsurgical results. Further osteotomies or osteoplasties are made after mobilization of the lower segment. The stabilization of the segment in its new position can be made with cortical wires, circumandibular wires, or plates and screws. The wound is irrigated and closed in two layers (muscle and mucosa) with resorbable suture.[2] 


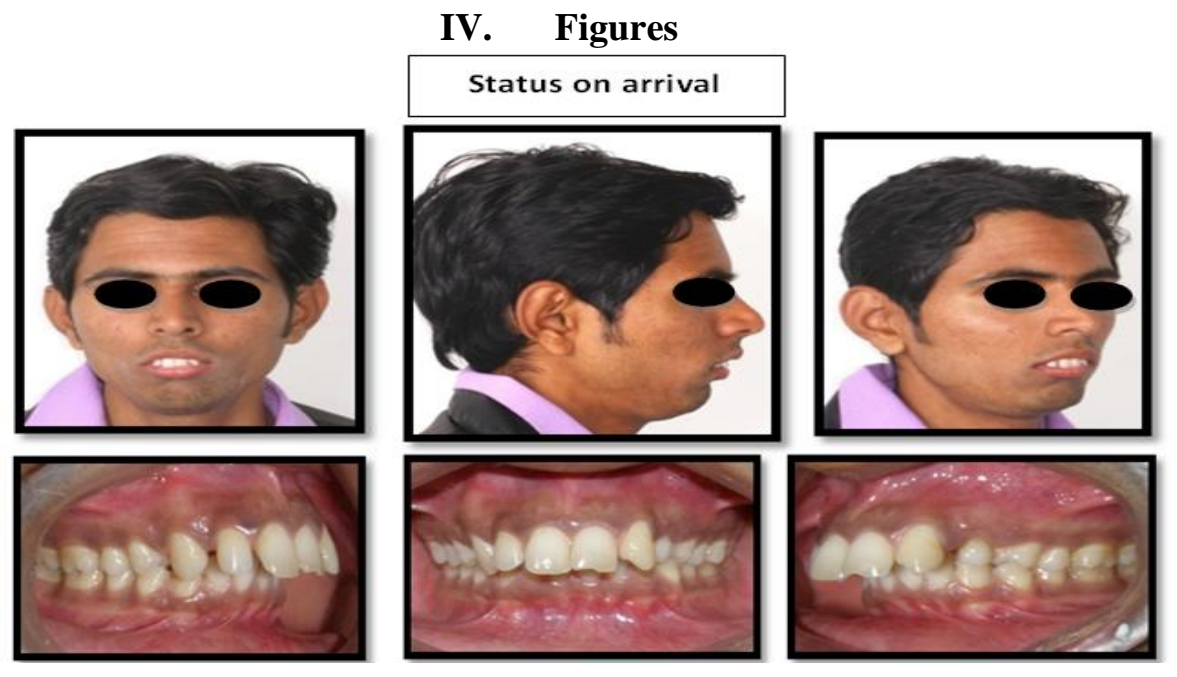

Presurgical orthodontics
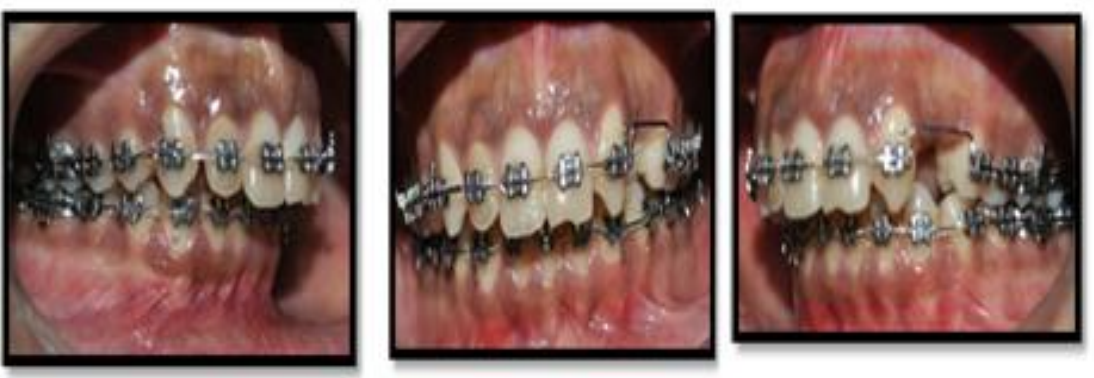

Surgical intervention
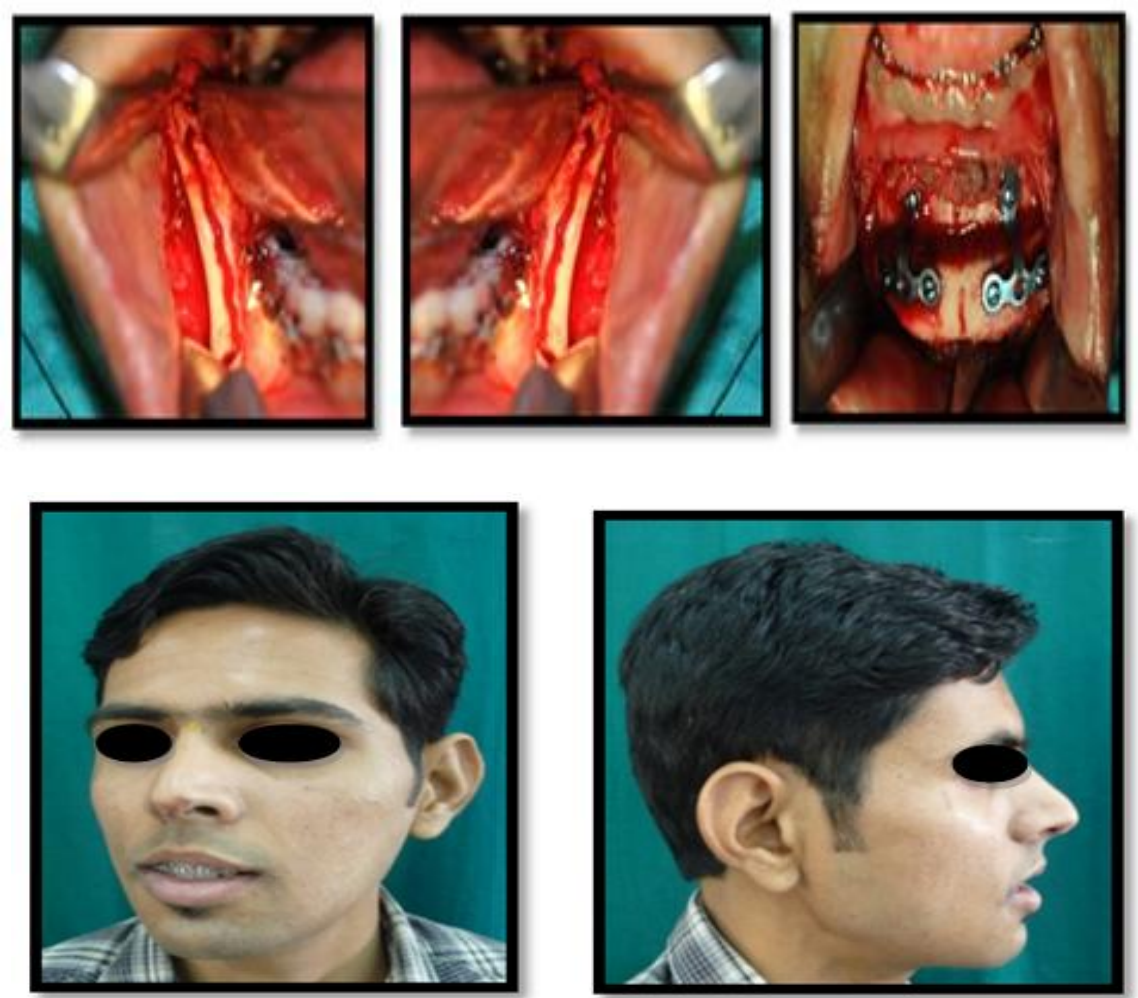

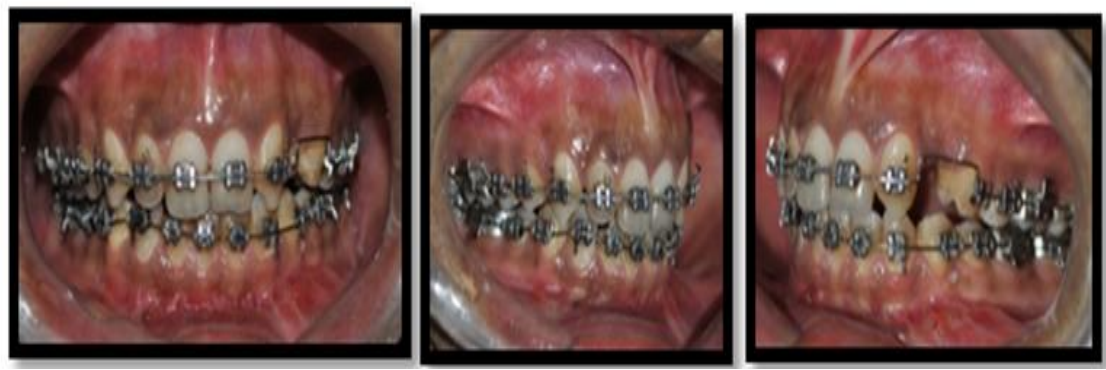

V. Discussion

Bilateral Sagittal Split Osteotomy is very popular and versatile procedure performed intraorally on mandibular body and ramus osteotomy and is an indispensable surgical procedure for the correction of lower jaw deformities [3]. Trauner and Obwegeser were the first to discuss its use in the English literature. Various complications are associated with this procedure like condylar positioning, malocclusion, unfavorable splits, relapse, nerve injury, TMJ dysfunction and hypomobility and hemorrhage [4]. Advantages of this procedure over the other osteotomy procedure are:-

1) It is an Intra-oral procedure which eliminates the extra-oral scar and hence more accepted by the patients.

2) Minimal alteration in the muscles of mastication.

3) Correction of obtuse gonial angle is possible during correction of skeletal class III deformities.

4) Broad bony healing ensures good healing of the ostetomised segments.

The procedure has done numerous modifications over time. Although the osteotomy is standardized, there is no agreement on the procedure for segment fixation, the selection of which depends on the surgeon's preference. No demonstrated technique has been proved to eliminate relapse completely.

\section{References}

[1]. Are there risk factors for osseous mandibular inferior border defects after bilateral sagittal split osteotomy?, Journal of CranioMaxillofacial Surgery(2017), doi: 10.1016/j.jcms.2016.12.015

[2]. Peterson's principles of oral and maxillofacial surgery, Second Edition

[3]. Bilateral Sagittal Split Osteotomy- A Case Report, Pravara Med Rev 2013;5(1)

[4]. Raymond J. Fonscea, Oral and Maxillofacial surgery Orthognathic surgery Vol 2 